\title{
Análise do desempenho do modelo WRF com alta resolução espacial na previsão de precipitação acumulada.
}

\author{
Performance analysis of the WRF model with high spatial resolution on cumulative \\ rainfall forecasting.
}

\author{
Luana Ribeiro Macedo*1, Rita de Cássia Marques Alves² , João Luiz Martins Basso², \\ Yoshihiro Yamasaki ${ }^{4}$
}

\footnotetext{
${ }^{1,2}$ Centro Estadual de Pesquisa em Sensoriamento Remoto e Meteorologia, CEPSRM/UFRGS

${ }^{3,4}$ Departamento da Faculdade de Meteorologia - UFPel
}

\begin{abstract}
Resumo
Eventos de precipitação intensa que ocorrem durante intervalos de tempo relativamente curtos, vêm ocorrendo com certa frequência no Brasil. Um dos Estados que têm sido muito afetado por esses eventos é o Rio Grande do Sul. No ano de 2013 a Região Metropolitana de Porto Alegre, em particular, foi assolada varias vezes por eventos desse tipo. Em especial o evento ocorrido no dia 11 de novembro na cidade de Porto Alegre, chegou até mesmo a registrar uma média de precipitação, em 24 horas, que superou a média da precipitação mensal. Esse evento causou inúmeros prejuízos econômicos e sociais, com inundações em inúmeros pontos da cidade, ocasionando a interrupção do transporte que empregam as linhas de trensurb e ônibus, resultados do grande volume de precipitação acumulado. A análise desse evento é realizada empregando os resultados dos processamentos do modelo de mesoescala WRF, configurado com grades de alta resolução espacial, bem como dados de observações das estações automáticas do INMET. Os resultados mostram que o modelo apresentou resultados consistentes e compatíveis com os valores das precipitações observadas, para o período de 24 e 48 horas, quando avaliados em relação às observações registradas em alguns pontos de observação.
\end{abstract}

Palavras-chave: precipitação intensa, WRF, mesoescala.

\begin{abstract}
Events of extremely intense rainfall which occurs during relatively short time span have been occurring with some frequency in Brazil. One of Brazilian state which has been strongly affected by these events is the Rio Grande do Sul. In 2013 the Metropolitan Region of Porto Alegre, in particular, has been devastated several times by events of this sort. In especial, the November $11^{\text {th }}$ event in the city of Porto Alegre, registered an average rainfall, in 24 hours, which exceeded the monthly average value. This event caused several economic and social losses, with flood over several locals over the city, giving rise to disruption of the trensurbs bus lines, due to the large volume of the accumulated precipitation. The analysis of this event is performed with the mesoescale model WRF processing, configured with high spatial resolution, as well as observational data of the INMET collected in the automatic weather stations network. The model results show a consistent and compatible values of precipitation compared with the observed values, for the 24 and 48 hours periods, evaluated with respect to the collected data in some points of observation.
\end{abstract}

Keywords: rainfall, WRF, mesoescale.

*lumeteorologia@gmail.com

Recebido: 13/03/2014 Revisado: 19/05/2014 Aceito: 13/03/2014 


\section{Introdução}

A precipitação é uma das variáveis meteorológica mais difícil de ser prevista, com precisão, pelos modelos numéricos. Essa dificuldade na destreza se deve à grande variabilidade dessa variável, tanto no espaço como no tempo, bem como à descontinuidade espacial e temporal. Segundo Smith et. al (1996) as atividades convectivas na atmosfera podem ser intensificadas pela topografia e a circulação local; que sob condições ambientais favoráveis podem gerar precipitação intensa e tempo severo em algumas horas.

A qualidade da previsão numérica tem uma grande dependência sobre os dados observados. Para a precipitação, em particular, há registros pontuais (coletadas em redes pluviométricas); bem como medidas indiretas, baseadas em técnicas de inferência empregando sensoriamento remoto (sensores a bordo de radar ou satélite). O sensoriamento remoto é a ferramenta mais adequada para obter estimativas de precipitação. A técnica que se utiliza permite realizar inferências sobre regiões onde não é possível obter dados pluviométricos de forma convencional, ou sobre regiões que, por uma razão ou outra, apresentam deficiências nas coberturas espaciais e temporais, como por exemplo, sobre os vastos oceanos e as imensas áreas continentais de difícil acesso.

Diversos centros de previsão do tempo vêm processando modelos globais de previsão do tempo, como o ECMWF (European Centre for Medium-Range Weather Forecasts) da comunidade Européia, NCEP (National Centers for Environmental Prediction) dos Estados Unidos, CPTEC (Centro de Previsão e Estudos Climáticos no Brasil. Entretanto, os modelos globais que vem sendo utilizados ainda apresentam deficiências, em particular, nas previsões da precipitação. Esta deficiência resulta, essencialmente, pela relativa pobre resolução espacial. Como consequência da incapacidade de incorporar inúmeras físicas da termo-hidrodinamica da atmosfera, de sub-escala espacial desses modelos, de forma mais compatível com o que ocorre na natureza não produzem previsões com grande destreza em prognósticos como a precipitação. Como a atmosfera está sujeita as características locais e aos fenômenos atmosféricos de pequena escala, como células convectivas, que não são bem representadas em modelos com baixa resolução espacial como nos modelos globais, torna-se imprescindível o emprego de modelos com maior resolução espacial. Borges et. al, (2008), afirmam que um fato que também contribui para o erro nas previsões é tecnológica, pois atualmente ainda não se dispõe de um sistema computacional rápido suficiente, com poder de processamento desejável, que permita aumentar a resolução espacial desses modelos e produzir previsões em tempo hábil. No entanto, já estão sendo desenvolvidos modelos de mesoescala que resolvem, parcialmente, esses problemas, Esses modelos são capazes de utilizar alta resolução espacial em seus processamentos e incorporar fenomenologias físicas, que permitem prever as alterações do tempo de uma forma mais fidedigna e em uma menor escala espacial.

As chuvas intensas podem ocorrer de forma isolada ou associada a outros sistemas meteorológicos. Geralmente elas são acompanhadas de trovoadas, descargas elétricas, granizos e ventos fortes e intensos. O maior índice de registros desses eventos na Região Sul do Brasil se deve às ocorrências no período das estações da primavera e verão, quando se tem maior ocorrências de vórtices ciclônicos e aglomerados convectivos na região. No ano de 2013 a Região Metropolitana de Porto Alegre (RMPA) foi atingida diversas vezes por eventos severos, com volumes de precipitação acima da média. Esses eventos têm preocupado muito a população, defesa civil, bem como os meteorologistas e profissionais que atuam com pesquisas sobre essa região.

O evento de precipitação na RMPA, que ocorreu no dia 11 de novembro de 2013, afetou bruscamente a cidade de Porto Alegre. Ele produziu intensidade de precipitação, em 24 horas, superior a média histórica registrada para o mês de novembro. Este evento causou transtornos não só para a capital gaúcha, como também em outros municípios da RMPA. Em algumas cidades houve a interrupção de energia elétrica, linhas de ônibus e trensurb foram suspensas, devido ao volume de chuva.

O sistema de modelagem numérica do tempo WRF (Weather Research Forecast), em especial, vem sendo empregado como uma das ferramentas necessárias para reproduzir, da melhor forma possível, a estrutura termo -hidrodinâmica da atmosfera. O presente trabalho foi realizado utilizando o modelo de mesoescala WRF para simular e verificar a habilidade do mesmo, em prever a ocorrência de precipitação, sobre a região Metropolitana de Porto Alegre, com ênfase na cidade de Porto Alegre, utilizando grades com alta resolução espacial. O desenvolvimento foi realizado como parte integrante de um projeto, de mestrado da primeira autora, cujo objetivo maior é analisar o impacto da assimilação de dados nos prognósticos do WRF.

\section{Dados e Metodologia}

A análise do evento da precipitação intensa ocorrida sobre a RMPA, no dia 11 de Novembro de 2012, foi procedida empregando imagens do canal infravermelho GOES - 13, dados da estação automática do INMET (Instituto Nacional de Meteorologia), cartas de superfície da marinha bem como os prognósticos do modelo WRF.

\section{1 Área de Estudo}

A área delimitada como foco principal no estudo compreende a Região Metropolitana de Porto Alegre (RMPA), também conhecida como Grande Porto Alegre, conforme ilustrada na Figura 1. Esta área engloba 33 municípios do estado do Rio Grande do Sul, que se encontra 
em intenso processo de conurbação. Ela representa uma extensão da capital Porto Alegre, constituindo com seus municípios lindeiros, uma vasta mancha urbana contínua, que inclui, também, os Vales dos Sinos e do Paranhana.

O relevo da Região Metropolitana apresenta planícies, suscetíveis a alagamentos ao longo da costa litorânea, bem como estepes e pradarias próximas da capital. As bacias fluviais que essa região apresenta e que, dependendo das intensidades da precipitação eventualmente causam problemas, engloba o rio Jacuí, que constitui a principal delas; os rios Gravataí, Sinos, Caí e Paranhana; além do lago Guaíba.

O clima desta região é classificado como subtropical úmido (Köppen e Geiger, 1928), tendo como característica marcante uma grande variabilidade temporal. A presença do lago Guaíba contribui à elevação da umidade atmosférica e alterar as condições termo-hidrodinâmicas da atmosfera na RMPA. As chuvas são relativamente bem distribuídas, sob o ponto de vista espacial e temporal, com a média anual da ordem de $1.300 \mathrm{~mm}$.
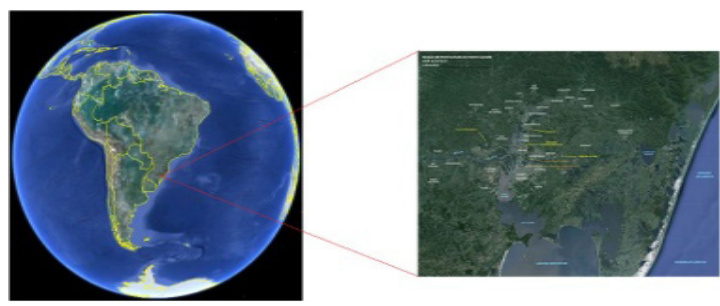

Figura 1. Localização da RMPA.

\subsection{Configuração do modelo WRF}

O modelo WRF, com núcleo constituído por equações compressível, Euleriano e não hidrostático foi configurado cobrindo quatro domínios de integração, conforme apresentados da figura 2 .

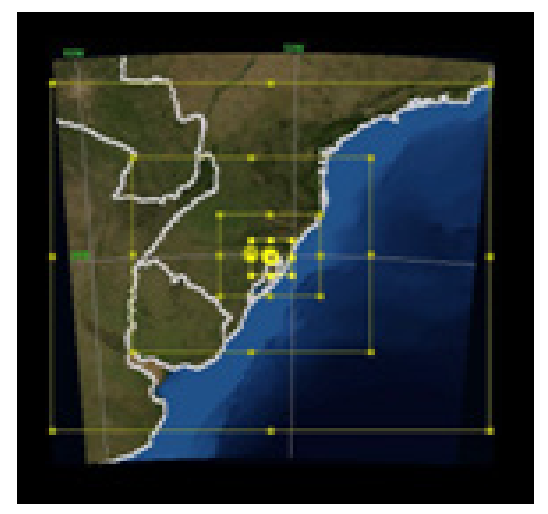

Figura 2. Domínios de Integração do modelo WRF

A resolução espacial, na horizontal, entre os pontos de grade e o numero de pontos de grade, de cada domínio de integração, são apresentados na tabela 1 .
Tabela 1. Domínios de Integração do modelo WRF.

\begin{tabular}{|c|c|c|}
\hline $\begin{array}{c}\text { Dominios de } \\
\text { Integracão }\end{array}$ & $\begin{array}{c}\text { Resolucão } \\
\text { Horizontal }\end{array}$ & $\begin{array}{c}\text { Pontos de } \\
\text { grade }\end{array}$ \\
\hline D1 & 18 & $115 \times 94$ \\
\hline D2 & 6 & $187 \times 157$ \\
\hline D3 & 2 & $238 \times 196$ \\
\hline D4 & 1 & $289 \times 256$ \\
\hline
\end{tabular}

O processamento do modelo WRF versão 3.5 foi feito utilizando, como condições iniciais e de contorno, os prognósticos do modelo global GFS (Global Forecast System) com formato GRIB2.

As parametrizações básicas configuradas, para o processamento do modelo WRF, foram as seguintes:

(a) Thompson et.al, para a o esquema de microfísica de nuvens - parametrização que geralmente é utilizada em processamentos com alta resolução espacial.

(b) RRTM (Rapid Radiative Transfer Model) - modelo de transferência radiativa que incluem múltiplas bandas espectrais bem como as microfísicas.

(c) Dudhia - que emprega uma ampla banda de ondas curta para se calcular a transferência radiativa, com uma simples integração que leva em consideração, tanto a absorção quanto o espalhamento da radiação, para condições de nuvens e céu claro.

(D) Mellor-Yamada-Janjic - que considera um esquema de prognóstico turbulento de energia cinética unidimensional com mistura vertical local.

(E) Kain-Fritsch - esquema de sub-grade de convecção prounda e superficial, utilizando uma abordagem de fluxo de massa descendente. Esse esquema

foi incorporado apenas ao primeiro domínio (D1), porque os outros apresentam resolução espacial inferior a $10 \mathrm{~km}$; por conseguinte a convecção foi tratada implicitamente. Detalhes sobre o modelo WRF, bem como a completa descrição sobre as parametrizações são apresentadas por Skamarock et al. 2008.

\section{Resultados e Discussões}

\subsection{Análise sinótica no dia do evento}

A imagem do canal infravermelho do satélite GOES - 13, do dia 11/11/2013 as 00 UTC (figura3), apresenta um sistema convectivo vindo da Argentina. Este sistema apresenta um núcleo com temperaturas próximas à $80^{\circ} \mathrm{C}$ em alguns pontos. $\mathrm{O}$ sistema prevaleceu, sobre o Estado do Rio Grande do Sul, durante todo o dia 11. Ele causou uma intensa precipitação, no período de 24 horas, cujo acumulado do dia, de $123 \mathrm{~mm}$, superou a média mensal dos últimos 10 anos onde o maior valor 
observado tinha sido de $72.1 \mathrm{~mm}$ (METSUL).

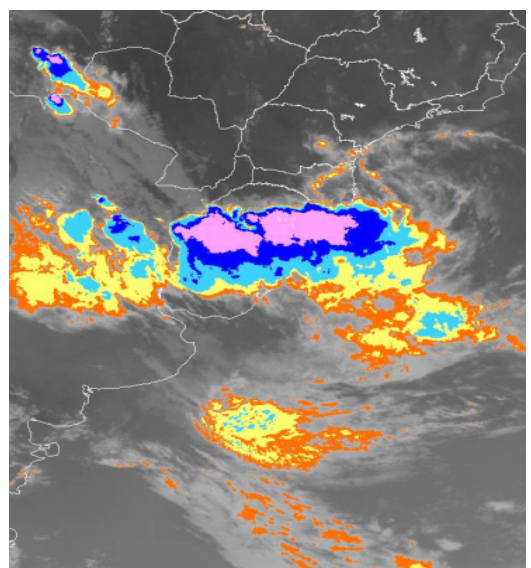

Figura 3. Imagem do satélite GOES - 13 às 00 UTC.

A carta sinótica de superfície, das 00UTC do dia 11 de Novembro de 2013, apresentada na figura 4, notase a atuação de um sistema de baixa pressão atuando sobre o Estado do Rio Grande do Sul. Este sistema, com valores inferiores a $1000 \mathrm{hPa}$, é consequência de um cavado em altos níveis, que produz o movimento do ar ascendente, causando também um aumento de nebulosidade na superfície.

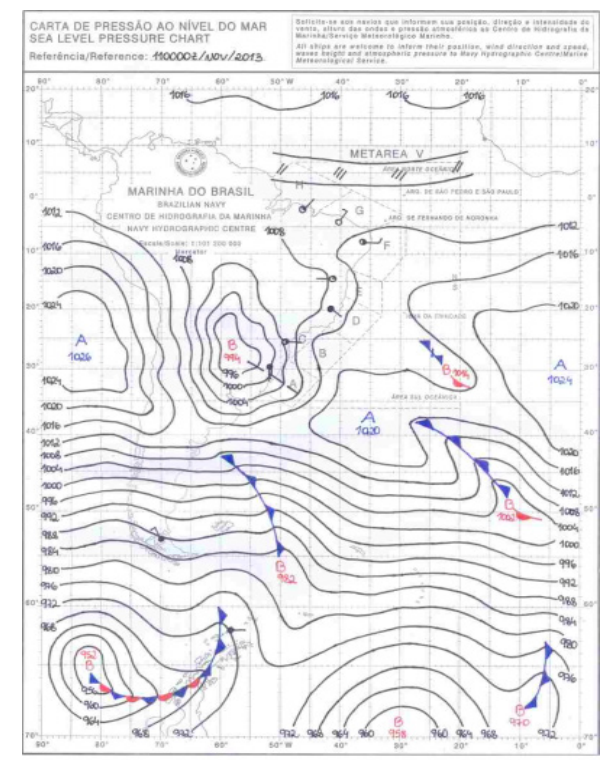

Figura 4. Carta de Superfície do dia 11/11/2013 as 00 UTC. Disponível em http://www.mar.mil.br.

\subsection{Análise dos Prognósticos do Modelo WRF}

Para realizar as simulações com o modelo WRF foi utilizado o cluster, do Centro Nacional de Supercomputação da Universidade Federal do Rio Grande do Sul, utilizando 36 processadores em paralelo.

Os resultados dos prognósticos das precipitações, obtidas nas simulações numéricas, foram comparados
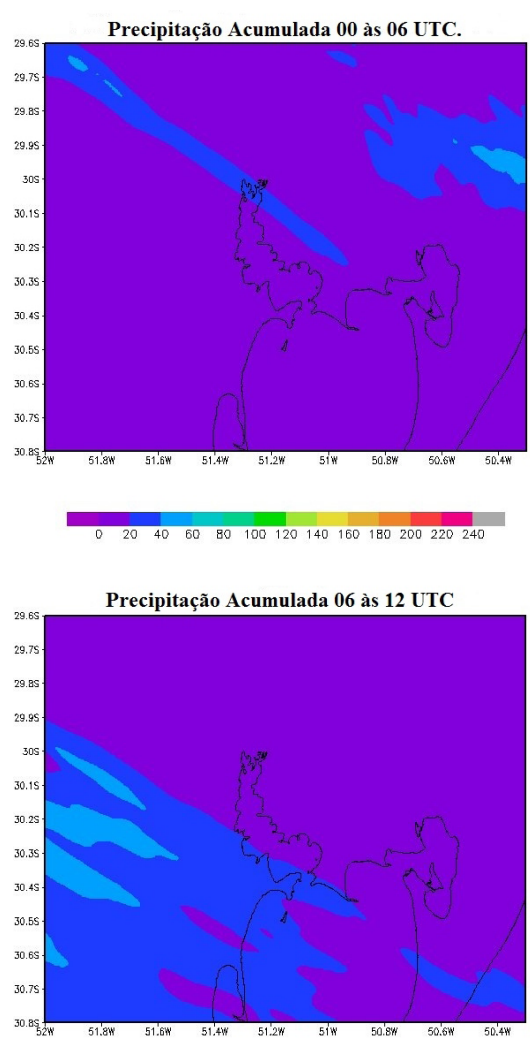

(b)
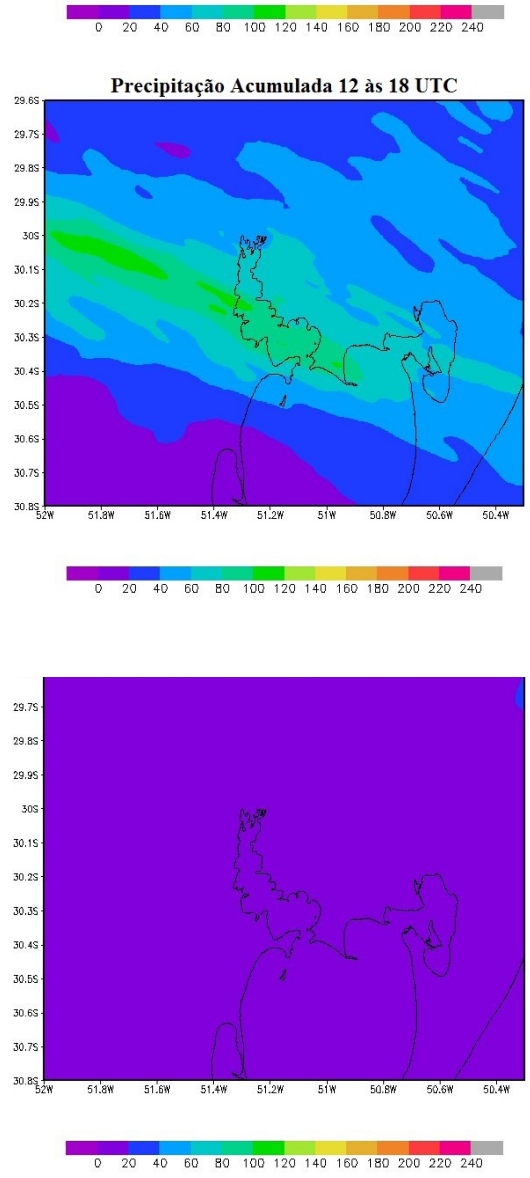

Figura 5. Precipitação acumulada de 6 em 6horas para o dia 11/11/2013. 
com os valores de precipitação pontuais da estação automática do INMET instalada na cidade de Porto Alegre. As simulações foram analisadas para o período de 6, 12, 24 horas do dia 11 e 48 horas do dia 10 à 11 de novembro para o domínio 4 , devido a sua melhor resolução espacial.

Na figura 5 é apresentado uma sequência de campos de precipitação acumulada de 6 em 6 horas. Elas caracterizam claramente um aumento da intensidade da precipitação com o tempo. Para as primeiras seis horas - as simulações do WRF - mostram a boa destreza no prognóstico da precipitação - com valores acumulados compatíveis com o que foi observado. Entretanto, nas seis horas subsequentes o modelo ora subestima os valores, ora superestima os valores da precipitação acumulada, conforme apresentado na Tabela 2.

Tabela 2. Comparação entre os acumulados de precipitação previsto e observado a cada 6 horas.

\begin{tabular}{|l|l|l|}
\hline & \multicolumn{2}{|c|}{ Precipitação } \\
$\begin{array}{c}\text { Horário } \\
\text { UTC }\end{array}$ & \multicolumn{2}{|c|}{ Mm } \\
\hline & WRF & INMET \\
\hline $00-06$ & 22 & 19.2 \\
\hline $06-12$ & 3.3 & 68.7 \\
\hline $12-18$ & 58.3 & 25 \\
\hline $18-00$ & 4.12 & 10.8 \\
\hline
\end{tabular}

Os campos da precipitação acumulada em 12 horas, prevista pelo modelo, apresentam menores valores, nas primeiras 12 horas e maiores nas 12 horas posteriores - conforme ilustrados na sequência das plotagens apresentadas na figura 6 - e os valores observados e apresentados na Tabela 3.

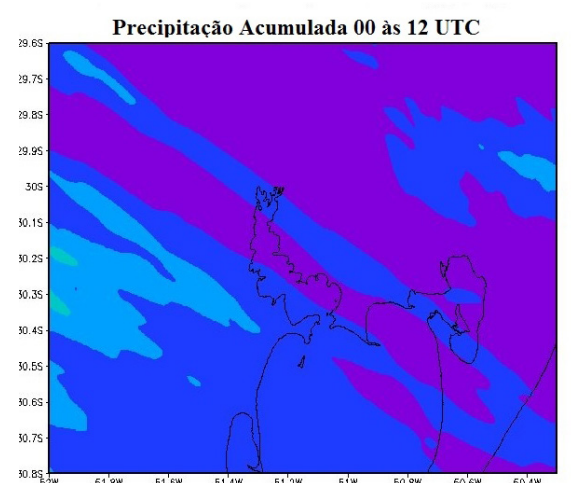

(a)

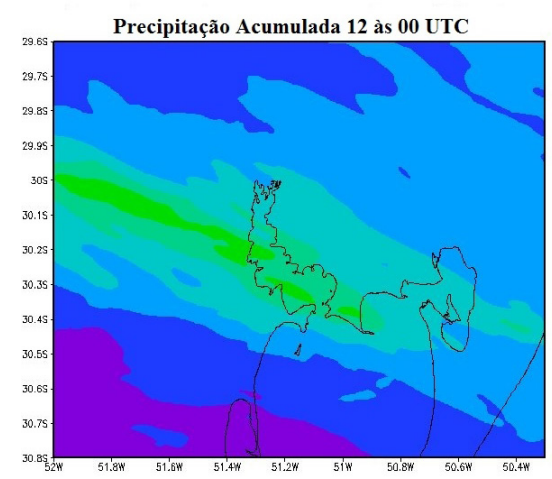

(b)

Figura 6. Precipitação acumulada de 12 em 12 horas para o dia 11/11/2013.

Tabela 3. Comparação entre os acumulados de precipitação previsto e observado a cada 12 horas.

\begin{tabular}{|l|l|l|}
\hline & \multicolumn{2}{|c|}{ Precipitação } \\
$\begin{array}{c}\text { Horário } \\
\text { UTC }\end{array}$ & \multicolumn{2}{|c|}{$\mathrm{mm}$} \\
\hline & WRF & INMET \\
\hline $00-12$ & 25.3 & 87.9 \\
\hline $12-00$ & 62.3 & 35.8 \\
\hline
\end{tabular}

Para a precipitação acumulada de 24 e 48 horas, embora o modelo não tenha apresentado valores tão próximo aos que foram observados, os resultados são relativamente satisfatórios. Isso, por que, o modelo conseguiu prognosticar, com destreza, o grande volume de chuva que se acumulou em Porto Alegre (vide Figura 7 e Tabela 4).

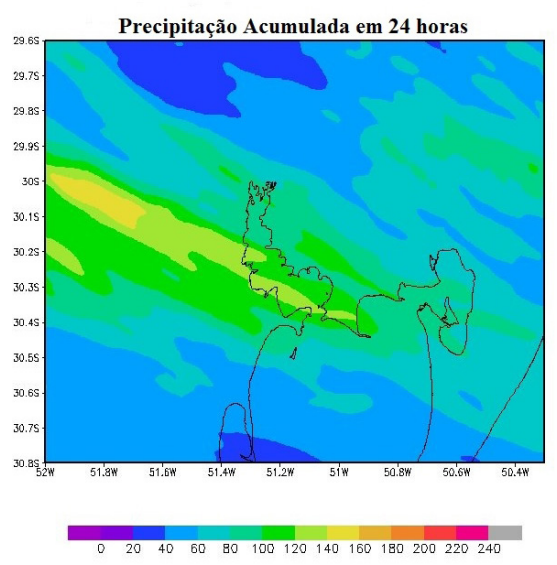

(a) 


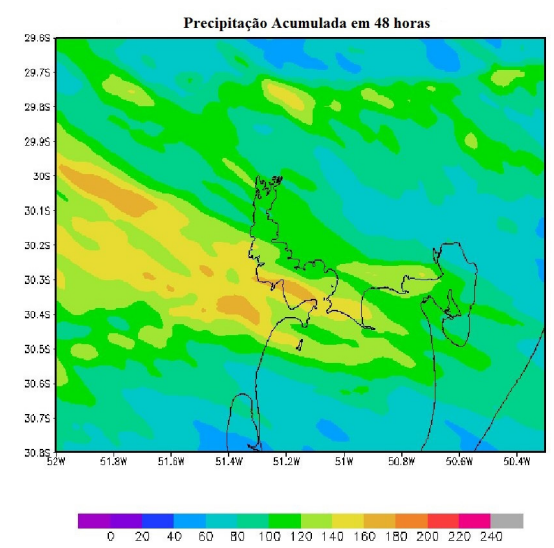

(b)

Figura 7. (a) Precipitação acumulada em 24 horas para o dia 11/11/2013. (b) Precipitação acumulada em 48 horas com inicio dia 10/11/2013.

Tabela 4. Comparação entre os acumulados de precipitação previsto e observado para 24 e 48 horas.

\begin{tabular}{|l|l|l|}
\hline & \multicolumn{2}{|c|}{ Precipitação } \\
$\begin{array}{c}\text { Horário } \\
\text { UTC }\end{array}$ & \multicolumn{2}{|c|}{ mm } \\
\hline & WRF & INMET \\
\hline $00-24$ & 88 & 123 \\
\hline $00-48$ & 105 & 135 \\
\hline
\end{tabular}

\section{Conclusões}

Segundo as análises realizadas, foi possível observar que a presença de um cavado em estágio incipiente e que se intensifica em altitude, é que induz a queda da pressão nos baixos níveis e é consequentemente responsável pelo sistema de baixa pressão em superfície - é que deixa a atmosfera instável e com muita nebulosidade, no Estado do Rio Grande do Sul. O sistema convectivo, vindo da Argentina, ao encontrar esta atmosfera instável é que acabou contribuindo para a ocorrência de precipitação intensa sobre a RMPA.

A indisponibilidade de dados observados com alta resolução espacial torna difícil o desenvolvimento de uma análise amiúde e completa sobre o prognóstico, com foco no evento severo ocorrido no dia 11 de Novembro de 2013. Para uma análise mais detalhada sobre o evento, foi utilizado o modelo WRF configurado com quatro domínios aninhados, cobrindo a região Sul do Brasil, e os parcos dados observacionais.

A análise apresentada foi feita com base no campo de precipitação prognosticada, para o domínio quatro (D4) do modelo WRF. Os resultados foram comparados com os dados pontuais de precipitação, coletadas pela estação automática do INMET, na cidade de Porto Alegre. O modelo WRF foi capaz de prever o grande volume de precipitação acumulada na cidade de Porto Alegre, no período de 24 e 48 horas. $\mathrm{O}$ acumulado da precipitação para intervalos de tempo menores o modelo não foi tão eficiente. Ele não foi capaz de prognosticar pontualmente os acumulados de 6 em 6 e 12 em 12 horas.

Na sequência dos desenvolvimentos, serão feitas novas análises e avaliações do evento intenso de precipitação explorado, considerando os módulos de assimilação de dados 3DVAR, com o WRF. Os processamentos serão executados, com o objetivo de se obter melhores condições iniciais e melhorar a qualidade da previsão para precipitação.

\section{Agradecimentos}

A CAPES pelo provimento da bolsa de estudos da primeira autora. E as demais instituições CPTEC, INMET, UCAR/NCAR, pela concessão dos dados para a realização do trabalho.

\section{Referências}

Borges, R.; Alexandrov V. ; Vas, J. J. del. ; Lumbreras, J. and Rodríguez, E. 2008: A comprehensive sensitivity analysis of the WRF model for air quality applications over the Iberian Peninsula, Atmospheric Environment, vol. 42, no. 37, pp. 8560-8574.

CPTEC. Disponível em: <http://www.cptec.inpe.br> Acesso em 25 nov. 2013

INMET. Disponível em: < http://www.inmet.gov.br> Acesso em 25 de nov. 2013

Köppen, W; Geiger,R. Klimate der Erd. Gotha: Verlag Justus Perthes 1928. Wall-map $150 \mathrm{~cm} \times 200 \mathrm{~cm}$.

METSUL. Disponível em: $<\underline{\text { http://www.metsul.com> }}$ Acesso em 20 de dez. 2013.

Skamarock, W.C.; et al., 2008: A Description of the Advanced Research WRF Version 3 . NCAR/TN 475+STR Tech. Note, UCAR,2008.

Smith, L. A. e I. Gilmour, 1998: Accountability and internal consistency in ensemble formation. from proceedings of the ecmwf workshop on predictability. The Proceedings of the ECMWF, ECMWF, ed., Workshop on Predictability, Reading, UK., 